\title{
Comparison of Nasal and Nasopharyngeal Swabs for Influenza Detection in Adults
}

\author{
Stephanie A. Irving, MHS; Mary F. Vandermause, BSMT; David K. Shay, MD, MPH; \\ and Edward A. Belongia, MD
}

\begin{abstract}
Objective: Examine differences in the detection of influenza by specimen and test type using paired nasal and nasopharyngeal swabs.
\end{abstract}

Design: Prospective study

Setting: Enrollment took place between January and March 2007 in a central Wisconsin population.

Participants: Adult patients were screened and enrolled by trained research coordinators following medical encounters for acute respiratory illnesses of $<10$ days duration.

Methods: Paired nasal and nasopharyngeal swabs were collected from consenting patients and tested by both real-time reverse transcriptase polymerase chain reaction (rRT-PCR) and viral culture. A composite measure of positivity was used as the gold standard; cases included any positive result by rRT-PCR or viral culture from either specimen type.

Results: Paired samples were collected from 240 adults; 33 (14\%) individuals tested positive for influenza by rRT-PCR. Using rRT-PCR, the sensitivity of the nasal swab was $89 \%$ ( $95 \% \mathrm{Cl}, 78 \%-99 \%)$ and the sensitivity of the nasopharyngeal swab was $94 \%$ ( $95 \% \mathrm{Cl}, 87 \%-100 \%)$, compared to a composite gold standard.

Conclusion: Test sensitivity did not vary significantly by swab type when using a highly sensitive molecular diagnostic test, but power was limited to detect modest differences.

Keywords: Detection; Influenza virus; Specimen type

I nfluenza is a major cause of acute respiratory illness worldwide and accounts for thousands of deaths in the United States in a typical season. ${ }^{1,2}$ The 2009 pandemic and increasing type-specific antiviral resistance have heightened the need for influenza testing that is accurate, timely, and well-tolerated by patients. ${ }^{3,4}$

A variety of specimens have been used for influenza testing including the nasopharyngeal (NP) swab, oropharyngeal swab, nasal wash, and nasal aspirate. The NP wash or aspirate is generally considered the 'gold standard' for virus isolation, but it is cumbersome to perform and unpleasant for patients. ${ }^{5-7}$

Corresponding Author: Stephanie Irving, MHS; Abt Associates; 2200 Century Parkway, Suite 950; Atlanta, GA 30345; Tel: 404.946.6309; Fax: 404.965.3065; E-mail: stephanie_irving@abtassoc.com
Swabs are easier and faster to collect and may be preferred by providers and patients. Comparative data on the sensitivity of influenza sampling procedures are limited; many studies focus on pediatric populations or use older diagnostic methods. ${ }^{5,8-12}$ Recent literature has focused on NP sampling compared to oropharyngeal or combined nose-throat swabs. ${ }^{6,7,10,12}$ To date, no studies have compared paired nasal and NP swabs collected from adults.

Several methods for laboratory diagnosis of influenza are available. Viral culture has historically been considered the 'gold standard' diagnostic test, but traditional culture can
Received: February 23, 2012

Revised: May I, 2012

Accepted: May 9, 2012

doi: $10.3|2| / \mathrm{cmr} .2012 .1084$

Financial Disclosure: Funding for this research was provided by a cooperative agreement with the Centers for Disease Control and Prevention, Atlanta, GA (I UOI Cl000I92-0I). 
require up to 7 days to obtain a positive result. ${ }^{5}$ Recent findings from studies utilizing real-time reverse transcriptase polymerase chain reaction (rRT-PCR) for the detection of respiratory pathogens suggest that the use of this current molecular technology may outweigh potential differences in sensitivity due to specimen type. . $^{710}$

We conducted a prospective study to examine differences in the detection of influenza by specimen type. Paired nasal and NP swabs were tested by both viral culture and rRT-PCR. Study procedures were reviewed and approved by the Marshfield Clinic Institutional Review Board, and all participants gave written informed consent for influenza testing.

\section{Methods}

\section{Participants}

Enrollment in the study took place between January and March 2007. Adult patients were enrolled by study staff following a medical encounter for acute respiratory illness. Eligible illnesses were $<10$ days duration and included fever, chills, or cough. ${ }^{13}$

\section{Clinical Specimens}

Both shallow nasal and NP swabs were collected from consenting patients. The NP swab was collected using an aluminum/plastic unishaft swab, inserted half the distance from the nares to the base of the ear, or to a depth of approximately 2 inches (Remel, Thermo Fisher Scientific, Lenexa, KS). ${ }^{14}$

As it was less invasive, the nasal swab was collected first, using a large tipped, plastic shafted Dacron swab. The swab was inserted approximately 1 centimeter, rubbed along the septum of the nostril for 3 to 5 seconds, and withdrawn. The swab was then placed in M4-RT viral transport media for testing. The more invasive NP swab was then collected using a smaller Dacron swab on a wire shaft. As per manufacturer's protocol, the swab was inserted to the point where the wire shaft meets the plastic sleeve, rotated, and withdrawn by scraping the septum. ${ }^{14}$ The wire-shafted swab was then placed in a separate M4-RT viral transport tube for testing. All samples were refrigerated for $<24$ hours until aliquots could be taken, which were then frozen until testing.
Diagnostic Testing

Real-time reverse transcriptase polymerase chain reaction (rRT-PCR) and viral cultures were performed at the Marshfield Clinic Research Foundation. rRT-PCR was performed on nucleic acid extracts from $200 \mu \mathrm{l}$ of clinical sample using the LightCycler Real-Time PCR System (Roche Diagnostics, Indianapolis, IN), and Invitrogen SuperScript III Platinum One-Step Quant RT-PCR chemistry (Life Technologies, Grand Island, NY). All rRT-PCR protocols, primers, and probes are property of and were provided by the Influenza Division of the Centers for Disease Control and Prevention (CDC) (protocols available from the $\mathrm{CDC}$ on request). ${ }^{15}$ The assay was a TaqMan based, real-time detection of the matrix 1 protein (M1) of influenza A and the non-structural protein 1 (NS1) of influenza B; the sequences of both proteins are highly conserved. The human RNase $\mathrm{P}$ gene primer and probe set served as an internal positive control for human nucleic acids.

Viral culture was performed using Madin-Darby canine kidney (MDCK) shell vial (Diagnostic Hybrid, Athens, OH). Cells were inoculated with $200 \mu \mathrm{l}$ of specimen and $1 \mathrm{ml}$ of culture media. Inoculated shell vials were centrifuged for one hour at $2000 \mathrm{rpm}$ to enhance viral contact and more rapid infection of the MDCK cells. The vials were incubated at $35^{\circ}$ to $37^{\circ} \mathrm{C}$. Cultures were microscopically examined daily for cytopathic effect (CPE). When CPE was observed or after 5 days of incubation with no $\mathrm{CPE}$, the shell vial monolayer was scraped, and a slide was prepared and stained for Influenza A and $\mathrm{B}$ immunofluorescent identification ( $\mathrm{D}^{3}$ Influenza $\mathrm{A} /$ Influenza B DFA Kit, Diagnostic Hybrid, Athens, OH).

\section{Statistical Analysis}

Sensitivities and 95\% confidence intervals (CI) were calculated from two-by-two tables. Sensitivity was calculated as compared to a composite gold standard, which included any positive result by rRT-PCR or viral culture from either specimen type. Sensitivities were compared using chi-square; $P$ values $<0.05$ were considered statistically significant. Data analysis was performed using SAS 9.2.

\section{Results}

Paired nasal and NP swabs were collected from 240 patients. The median age of the patients was 60 years (range 47 to 91

Table 1. Comparison of the sensitivity of nasal and nasopharyngeal swab specimens for the detection of influenza by viral culture and rRT-PCR.*

\begin{tabular}{|c|c|c|c|c|c|c|c|}
\hline & \multicolumn{3}{|c|}{ Nasal swabs } & \multicolumn{3}{|c|}{ Nasopharyngeal swabs } & \multirow[b]{2}{*}{$P$ value } \\
\hline & $\begin{array}{l}\text { No. } \\
\text { positive } \\
\text { samples }\end{array}$ & $\begin{array}{c}\text { Sensitivity } \\
(\%)\end{array}$ & $95 \% \mathrm{Cl}$ & $\begin{array}{l}\text { No. } \\
\text { positive } \\
\text { samples }\end{array}$ & $\begin{array}{c}\text { Sensitivity } \\
(\%)\end{array}$ & $95 \% \mathrm{Cl}$ & \\
\hline Viral culture & 14 & 40.0 & $23.8-56.2$ & 18 & 51.4 & $34.9-68.2$ & 0.34 \\
\hline rRT-PCR & 31 & 88.6 & $78.0-99.1$ & 33 & 94.3 & $86.6-100$ & 0.40 \\
\hline \multicolumn{8}{|c|}{$\begin{array}{l}\text { *Sensitivity calculated as compared to a composite gold standard. Gold standard cases included any positive result by rRT-PCR or viral culture from either } \\
\text { specimen type }(n=35) \text {. } \\
\text { †Sensitivity of nasal swab as compared to NP swab, using same diagnostic testing method. }\end{array}$} \\
\hline
\end{tabular}


years), 151 (63\%) were female, 147 (61\%) were vaccinated with the 2006-2007 influenza vaccine, and 109 (45\%) had a chronic medical condition. The mean interval from symptom onset to specimen collection was 4.8 (range 0-10) days.

A total of 35 swabs tested positive for influenza by either rRTPCR or viral culture by either swab type (CGS). Thirty-one samples were positive for influenza $A$; four samples were positive for influenza B. There were $14(6 \%)$ nasal swabs and $18(8 \%)$ NP swabs that tested positive for influenza by viral culture. There were 31 (13\%) nasal swabs and 33 (14\%) NP swabs that tested positive for influenza by rRT-PCR. The nasal swab had $40.0 \%$ sensitivity by viral culture and $88.6 \%$ sensitivity by rRT-PCR, when calculated compared to the CGS $(P<0.0001)$. The NP swab had $51.4 \%$ sensitivity by viral culture and $94.3 \%$ sensitivity by rRT-PCR, compared to the CGS $(P<0.0001)$. The sensitivity differences by swab type when using the same diagnostic test were not significant (table 1).

Of the $18 \mathrm{NP}$ swabs positive for influenza by viral culture, 14 paired nasal swabs tested positive, for a sensitivity of $77.8 \%$ (95\% CI, 58.6\%-97.0\%) for the nasal swab, as compared to the NP swab. Of the 33 NP swabs positive by rRT-PCR, 29 paired nasal swabs tested positive, for a sensitivity of $87.9 \%$ (95\% CI, 76.7\%-99.0\%) for the nasal swab, as compared to the NP swab. The difference in sensitivity of the nasal swab as compared to the paired NP swab by diagnostic test was not significant $(P=0.35)$.

\section{Discussion}

This study compared the sensitivities of two specimen collection methods using two diagnostic methods for the diagnosis of influenza. The nasal swab was less sensitive than the NP swab, irrespective of diagnostic test, but the difference in sensitivities between sampling methods was not significant. Real-time RT-PCR was significantly more sensitive than viral culture, irrespective of specimen collection method.

Our results, together with findings from the literature, suggest that less invasive methods of specimen collection may be acceptable in the era of molecular testing. A larger study of influenza detection using combined nose and throat swabs versus NP aspirates found that the combined swabs had a higher diagnostic yield, but the performance of nasal swabs alone was not evaluated. ${ }^{7}$ A recent large pediatric study reported $88 \%$ sensitivity for the detection of influenza A using NP aspirates, and $84 \%$ sensitivity with the nasal swab when tested by PCR; the difference in sensitivities was not statistically significant $(P=0.72) .{ }^{12}$ Lambert and colleagues ${ }^{10}$ compared combined nose-throat swabs with NP aspirates in a large pediatric population. Reported sensitivities of the nosethroat swab were $92 \%$ for the detection of influenza $\mathrm{A}$ and $100 \%$ for influenza B. ${ }^{10}$

We are not aware of any other published studies evaluating paired nasal and NP swabs from adults using rRT-PCR as the diagnostic method for detection of influenza. A strength of our study is the use of consistent recruitment procedures and standardized sample collection methods. The most important limitation of the study is the limited power to detect modest differences in sensitivity; only 35 participants tested positive for influenza. Sensitivity of sample collection methods may vary by influenza type/subtype, and our case numbers did not allow for stratified analyses. The paired specimens in this analysis were collected from older adults only; thus, we are unable to generalize our results to younger populations. Finally, specimens underwent an additional freeze-thaw cycle between rRT-PCR and culture testing. While this has the potential to affect the sensitivity of the viral culture, any impact would have been minor. Additionally, the focus of our investigation was the agreement between collection methods, not diagnostic test, as literature has previously demonstrated increased sensitivity of PCR compared to viral culture.

\section{Conclusion}

Traditional specimen collection methods for the detection of influenza are based on the use of viral culture as the diagnostic test. ${ }^{5}$ A 'gold standard' sampling method has not been identified and validated for influenza detection using rRTPCR, but emerging evidence suggests that less invasive samples may have comparable sensitivity to nasopharyngeal swabs or aspirates when using molecular diagnostic tests.

\section{Acknowledgments}

The authors would like to thank LaShondra Berman, Steve Lindstrom, Jennifer Meece, Marshfield Clinic Research Foundation Core Lab staff, Epidemiology Research Center research coordinators, and 2006-2007 Influenza Vaccine Effectiveness study participants for their contributions to this project.

The findings and conclusions in this report are those of the authors and do not necessarily represent the official position of the Centers for Disease Control and Prevention.

\section{References}

1. Dushoff J, Plotkin JB, Viboud C, Earn DJ, Simonsen L. Mortality due to influenza in the United States--an annualized regression approach using multiple-cause mortality data. Am J Epidemiol 2006;163:181-187.

2. Thompson WW, Weintraub E, Dhankhar P, Cheng PY, Brammer L, Meltzer MI, Bresee JS, Shay DK. Estimates of US influenza-associated deaths made using four different methods. Influenza Other Respi Viruses 2009;3:37-49.

3. Dutkowski R. Oseltamivir in seasonal influenza: cumulative experience in low- and high-risk patients. J Antimicrob Chemother 2010;65:ii11-ii24.

4. Ferguson NM, Cummings DA, Fraser C, Cajka JC, Cooley PC, Burke DS. Strategies for mitigating an influenza pandemic. Nature 2006;442:448-452.

5. Agoritsas K, Mack K, Bonsu BK, Goodman D, Salamon D, Marcon MJ. Evaluation of the Quidel QuickVue test for detection of influenza A and $\mathrm{B}$ viruses in the pediatric emergency medicine setting by use of three specimen collection methods. J Clin Microbiol 2006;44:2638-2641. 
6. Lieberman D, Lieberman D, Shimoni A, Keren-Naus A, Steinberg R, Shemer-Avni Y. Identification of respiratory viruses in adults: nasopharyngeal versus oropharyngeal sampling. J Clin Microbiol 2009;47:3439-443.

7. de la Tabla VO, Masiá M, Antequera P, Martin C, Gazquez G, Buñuel F, Gutiérrez F. Comparison of combined nose-throat swabs with nasopharyngeal aspirates for detection of pandemic influenza A/H1N1 2009 virus by real-time reverse transcriptase PCR. J Clin Microbiol 2010;48:3492-3495.

8. Heikkinen T, Marttila J, Salmi AA, Ruuskanen O. Nasal swab versus nasopharyngeal aspirate for isolation of respiratory viruses. J Clin Microbiol 2002;40:4337-4339.

9. Heikkinen T, Salmi AA, Ruuskanen O. Comparative study of nasopharyngeal aspirate and nasal swab specimens for detection of influenza. BMJ 2001;322:138.

10. Lambert SB, Whiley DM, O'Neill NT, Andrews EC, Canavan FM, Blethly C, Siebert DJ, Sloots TP, Nissen MD. Comparing nose-throat swabs and nasopharyngeal aspirates collected from children with symptoms for respiratory virus identification using real-time polymerase chain reaction. Pediatrics 2008;122:e615-e620.

11. Schmid ML, Kudesia G, Wake S, Read RC. Prospective comparative study of culture specimens and methods in diagnosing influenza in adults. BMJ 1998;316:275.

12. Sung RY, Chan PK, Choi KC, Yeung AC, Li AM, Tang JW, Ip M, Tsen T, Nelson EA. Comparative study of nasopharyngeal aspirate and nasal swab specimens for diagnosis of acute viral respiratory infection. J Clin Microbiol 2008; 46:3073-3076.

13. Belongia EA, Kieke BA, Donahue JG, Greenlee RT, Balish A, Foust A, Lindstrom S, Shay DK; Marshfield Influenza Study Group. Effectiveness of inactivated influenza vaccines varied substantially with antigenic match from the 2004-2005 season to the 2006-2007 season. J Infect Dis 2009; 199:159-167.

14. Cadham Provincial Laboratory Public Health. Untitled. Available at: http://www.gov.mb.ca/health/publichealth/cpl/ docs/nasopharyngeal_collection.pdf. Accessed April 26, 2012.

15. World Health Organization. CDC Protocol of Realtime RTPCR for Swine Influenza A(H1N1). April 28, 2009. Available at: http://www.who.int/csr/resources/publications/swineflu/ CDCrealtimeRTPCRprotocol_20090428.pdf. Accessed April 26, 2012.

\section{Author Affiliations}

Stephanie A. Irving, MHS, ${ }^{*}+$; Mary F. Vandermause, BSMT; David K. Shay, MD, MPHs ; Edward A. Belongia, MD*

"Epidemiology Research Center, Marshfield Clinic Research Foundation, Marshfield, Wisconsin, USA

${ }^{\dagger}$ Current Affiliation: Senior Analyst, Abt Associates, Atlanta, Georgia, USA

*ore Laboratory, Marshfield Clinic Research Foundation, Marshfield, Wisconsin, USA

Influenza Division, Centers for Disease Control and

Prevention, Atlanta, Georgia, USA 